\title{
TVT Y TOT PARA LA CORRECCIÓN QUIRÚRGICA DE LA INCONTINENCIA FEMENINA DE STRESS. COMPARACIÓN DE AMBAS TÉCNICAS
}

\author{
G. Torres Zambrano, M. Lujan Galán', C. Martín García, A. García Tello, N. Rodríguez \\ García y A. Berenguer Sánchez.
}

Servicio de Urología. Hospital Universitario de Getafe. Madrid y Servicio de Urología'. Hospital Infanta Cristina. Madrid. España.

\begin{abstract}
Resumen.- OBJETIVO: Evaluar los resultados en nuestras manos con las técnicas TVT y TOT.

MÉTODOS: Análisis retrospectivo de pacientes con incontinencia urinaria de esfuerzo operadas en nuestro servicio, mediante las técnicas de TVT y TOT. Evaluamos la continencia postoperatoria, considerando a las pacientes curadas si presentaban continencia total y como fracaso a cualquier tipo de pérdida involuntaria con el esfuerzo, independientemente de su severidad, registrándose el momento de su aparición. Para ello se empleó un análisis Kaplan-Meier comparando ambas técnicas mediante test de Breslow. Además se evaluaron las complicaciones postoperatorias.
\end{abstract}

RESULTADOS: El análisis se realizó con 128 pacientes intervenidas mediante TVT 169 pacientes, 53,9\%) y TOT 159 pacientes, 46, 1\%). La media de edad para el TVT fue de 54,4 años (IC 95\% 52,0 - 56,8), y de 59 años para el TOT (IC 95\% 55,9-62,1). El tiempo medio de seguimiento fue 18,7 meses (IC 95\% 15,6-21,9) para el TVT y 7,4 meses (IC 95\% 5,8-8,9) para TOT. La tasa global de continencia fue de $86,7 \%$, siendo de $88,4 \%$ para TVT y $84,7 \%$ para TOT. La probabilidad de permanecer continente a partir de los 6 meses de la cirugía fue de $89,1 \%$ para el TVT y de 78,2\% para el TOT (diferencia no significativa, $p=0,31$ ), con la práctica totalidad de los fracasos antes de los 6 meses postcirugía. Con el TVT se produjo mayor sintomatología de urgencia $(33,3 \%, p=0,001)$ e incontinencia de urgencia $(18,8 \%, p=0,16)$, retención de orina $(11,6 \%$, $p=0,38)$, y dos pacientes con hematoma prevesical que requirieron la retirada de la malla. Se observó mayor dolor postoperatorio con el TOT (20,3\%, p=0,005).

CONCLUSIONES: Nuestros resultados evidencian una eficacia similar con ambas técnicas, presentándose los fracasos durante los primeros meses tras el procedimiento. Las complicaciones tipo urgencia miccional son significativamente mayores con el TVT.

Palabras clave: Incontinencia urinaria de esfuerzo. Cirugía de incontinencia. Complicaciones quirúrgicas.

Summary.- OBJECTIVES: Retrospective analysis of a series of patients with stress urinary incontinence that underwent surgery in our department, by means of the TVT and TOT techniques. We evaluate post operative continence, considering "cured" the patient with complete continence and "failure" any type of involuntary stress 
urine leak, independently of its severity, registering the time of its appearance. For this purpose we used the Kaplan-Meier analysis comparing both techniques with the Breslow test. Additionally, postoperative complications were evaluated.

RESULTS: 128 patients who underwent surgery by TVT (69 patients, 53.9\%) and TOT (59 patients, 46.1\%). Mean age was 54.4 years for TVT $195 \%$ Cl 52.0-56.8) and 59 years for TOT $195 \% \mathrm{Cl}$ 55.9-62.11. Mean follow-up time was 18.7 months for TVT 195\% Cl 15.6$21.9)$ and 7.4 months for TOT $(95 \% \mathrm{Cl}$ 5.8-8.9). Overall continence rate was $86.7 \%, 88.4 \%$ for TVT and $84.7 \%$ for TOT. The probability of being continent six months after surgery was $89.1 \%$ for TVT and $78.2 \%$ for TOT (no significant differences, $p=0.31$ ), with almost all failures within the first six months after surgery. TVT was more frecuently associated with urgency symptoms $133.3 \%, p=0.001)$ and urge incontinence $(18.8 \%, p=$ $0.16)$, urinary retention $(11.6 \%, p=0.38)$, and prevesical hematoma requiring mesh retrieval. Greater post operative pain was observed with TOT $120.3 \%$, p= 0.005)

CONCLUSIONS: Our results evidence a similar efficacy with both techniques, with the failures appearing over the first months after surgery. Complications of the voiding urgency type are significantly more frequent with TVT.

Keywords: Stress urinary incontinence. Incontinence surgery. Surgical complications.

\section{INTRODUCCIÓN}

La incontinencia urinaria tiene un gran impacto sobre la calidad de vida de las personas que la padecen, por ello consideramos prioritario la resolución de esta patología. En España la prevalencia es de dos millones de personas, y de estas un $50 \%$ son mujeres mayores de 60 años (1). La Sociedad Internacional de Continencia ha definido la incontinencia urinaria de esfuerzo como "cualquier pérdida de orina involuntaria" (2). Para el tratamiento de la misma, se han descrito diferentes técnicas quirúrgicas desde la invención de la plicatura uretral descrita por Kelly y Dumm en 1914 (3), hasta nuestros días, en los que se buscan procedimientos mínimamente invasivos (4).

A mediados de los noventa se describieron técnicas para la suspensión de la uretra media y restitución del ángulo uretrovesical sin tensión. El primero fue el TVT (Tension-Free Vaginal Tape) descrito por
Ulmsten y cols. (5), que consiste en el paso de una malla de polipropileno a través de la fascia endopélvica. Sin embargo, se han descrito lesiones intestinales, vasculares, vesicales y perforaciones uretrales debido a la técnica empleada $(6,7)$. Posteriormente Delorme y cols. (8) diseñaron el TOT (Transobturator Tape), en un intento de evitar las perforaciones vesicales, al pasar la malla a través del agujero obturador, disminuyendo además el tiempo quirúrgico, al no requerir la realización de cistoscopia (9). Ambas técnicas presentan resultados muy similares, si bien se han descrito mas complicaciones con el TVT.

El objetivo del presente estudio es evaluar los resultados retrospectivos en nuestras manos con las técnicas TVT y TOT, comparando su eficacia y las complicaciones derivadas de los mismos.

\section{MATERIALES Y MÉTODOS}

Se llevó a cabo un análisis retrospectivo de 128 mujeres operadas por primera vez por presentar incontinencia urinaria de esfuerzo, mediante las técnicas de TVT y TOT. Las pacientes fueron interrogadas y exploradas en la consulta por el mismo urólogo, excluyendo aquellas que presentaran prolapsos genitales. Se ofreció el TVT durante el periodo comprendido entre octubre del 2002 y abril del 2006, y TOT desde abril del 2005 hasta la fecha. Para facilitar el análisis de los resultados, dividimos los mismos en varios intervalos de tiempo, de 6 en 6 meses hasta completar los 2 años de seguimiento.

Evaluamos la continencia postoperatoria a lo largo del seguimiento, considerando a las pacientes como curadas si presentaban continencia total y como fracaso a cualquier tipo de pérdida involuntaria con el esfuerzo, independientemente de su severidad, registrándose el momento de su aparición, así como la proporción de pacientes libre de la misma en cada intervalo de seguimiento $(10,11)$. Para ello se empleó un análisis Kaplan-Meier comparando ambas técnicas mediante test de Breslow (12).

Además examinamos las complicaciones postoperatorias presentadas en nuestra muestra, haciendo una comparación entre las dos técnicas estudiadas incluyendo dolor postoperatorio, la urgencia, incontinencia de urgencia, retención postoperatoria e infección de la herida quirúrgica.

\section{RESULTADOS}

Durante el periodo comprendido entre, octubre de 2002 y diciembre de 2007, se estudiaron 
TABLA I. PROBABILIDAD DE PERMANECER LIBRE DE INCONTINENCIA AL FINAL DEL INTERVALO.

\begin{tabular}{|c|c|c|c|c|c|c|}
\hline & \multicolumn{5}{|c|}{ Inicio del intervalo } \\
\hline & & 0 & 6 & 12 & 18 & 24 \\
\hline \multirow{4}{*}{$\begin{array}{l}\text { TIPO DE } \\
\text { CIRUGIA }\end{array}$} & Pacientes evaluables & 69 & 52 & 37 & 28 & 22 \\
\hline & $\begin{array}{l}\text { Probabilidad continencia al } \\
\text { final del intervalo }\end{array}$ & 891 & .891 & 891 & 891 & 891 \\
\hline & Pacientes evaluables & 59 & 26 & 9 & 3 & 1 \\
\hline & $\begin{array}{l}\text { Probabilidad continencia al } \\
\text { final del intervalo }\end{array}$ & 828 & .782 & .782 & .782 & .782 \\
\hline
\end{tabular}

128 mujeres intervenidas quirúrgicamente con incontinencia urinaria de esfuerzo, de estas, a 69 se les practicó TVT $(53,9 \%)$ y a las 59 restantes TOT $(46,1 \%)$. La media de edad fue de 54,4 años para el TVT (IC 95\% 52,0 - 56,8), con rango entre 34, 1 y 78,3 años. Para el TOT se observó una media de 59 años (IC 95\% 55,9-62,1), y un rango entre 35,9 y 78,7 años. El tiempo medio de seguimiento fue 18,7 meses (IC 95\% 15,6-21,9) para el TVT y para TOT 7,4 meses (IC 95\% 5,8-8,9).

\section{Evaluación de la continencia a lo largo del seguimiento:}

Ambas técnicas mostraron buenos resultados, teniendo tasas globales de continencia de $86,7 \%$, e individuales de $88,4 \%$ para TVT y $84,7 \%$ para TOT. Se presentaron 17 eventos de fracaso en la serie, de ellas 8 entre los TVT $(11,6 \%)$ y 9 entre los TOT $(15,3 \%)$. La probabilidad de permanecer continente a partir de los 6 meses de la cirugía fue de $89,1 \%$ para el TVT y de $78,2 \%$ para el TOT (diferencia no significativa, $p=0,31)$, con la práctica totalidad de los fracasos antes de los 6 meses postcirugía (Tabla l).

En la Figura 1 representamos la evolución de la continencia postoperatoria en la muestra global estudiada ( $\mathrm{N}=128)$.

Observamos una diferencia no significativa $(p=0,31)$ a favor del TVT en cuanto a resultados en términos de continencia, con un $89,1 \%$ totalmente continentes a los 2 años de seguimiento (Figura 1).

\section{Evaluación de complicaciones:}

En la Tabla II se hace una comparación de las complicaciones encontradas con las dos técnicas empleadas.

En nuestra serie, con el TVT se produjo mayor sintomatología de urgencia postoperatoria $(33,3 \%$, $p=0,001)$, incontinencia de urgencia $(18,8 \%, p=$ $0,16)$ y retención de orina $(11,6 \%, p=0,38)$. Además, dos pacientes sometidas a corrección mediante esta técnica presentaron hematoma prevesical que requirió la retirada de la malla de polipropileno (parcial en un caso y total en otro). Se observó mayor dolor postoperatorio en las pacientes intervenidas mediante TOT $(20,3 \%, p=0,005)$.

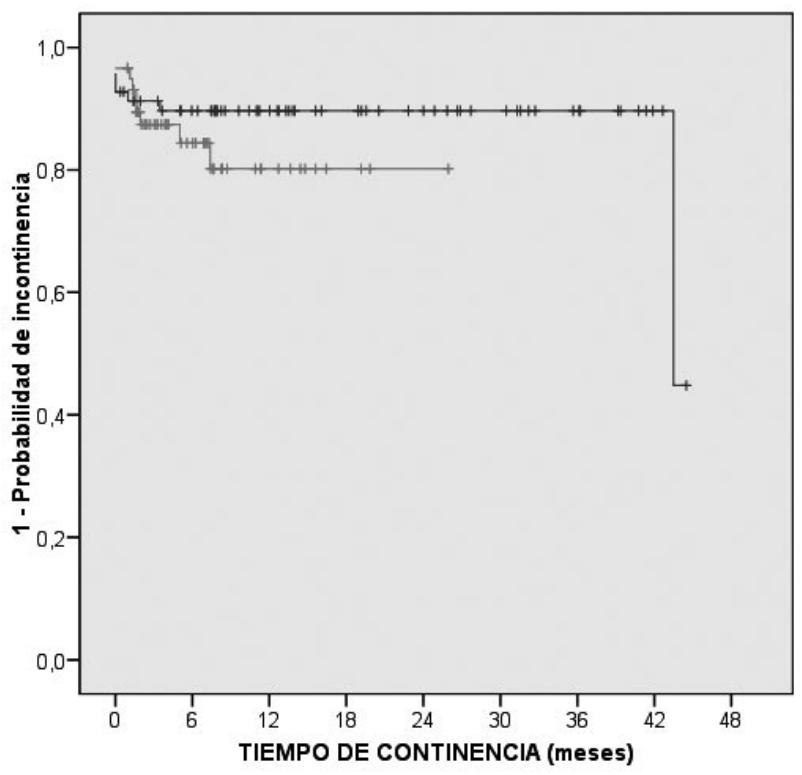

TIPO DE CIRUGIA

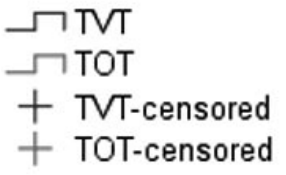

FIGURA 1. Evolución de la continencia postoperatoria en la muestra global estudiada ( $N=128)$. 


\section{DISCUSIÓN}

Realizamos un estudio comparativo retrospectivo con 128 mujeres con incontinencia de esfuerzo operadas por primera vez, para evaluar los resultados en nuestras manos de las técnicas TVT y TOT, así como las complicaciones presentadas a lo largo del seguimiento.

Encontramos una diferencia no significativa a favor del TVT $(p=0,5)$, en términos de continencia, obteniendo en general buenos resultados con ambas técnicas $(86,7 \%$ de continencia de la serie). Observamos que los fracasos en ambos procedimientos se dan durante el primer intervalo de tiempo que consideramos en nuestro estudio (de 0 a 6 meses), posterior a esto, no hay nuevos episodios que desvíen la curva se supervivencia libre de incontinencia de una manera importante, lo que nos hace considerar ambas técnicas como efectivas tras pasar dicho intervalo.

Datos similares han sido publicados por Ulmsten, después de un estudio de 45 pacientes seguidas por un periodo de 3 años, en el que los resultados no empeoraron con el tiempo y las tasas de curación fueron del 90\% (13). DeTayrac publicó en el 2003 un estudio comparativo entra ambas técnicas, teniendo tasas de curación para TVT de $83,4 \%$ y $90 \%$ para TOT, sin encontrar diferencias estadísticamente significativas (6). Para Liapis y cols las tasas de curación fueron del $89 \%$ para TVT y $90 \%$ para TOT (14). Mellier y cols. reportaron tasas de $90 \%$ para TVT y $95 \%$ para TOT (15), Darai y cols de $89.3 \%$ y $88,6 \%$ para TVT y TOT respectivamente (9). Además, parece existir una tendencia a tener mayores tasas de fracaso asociadas a mayor edad de las pacientes y a una disminución de la fisiología normal uretral por una deficiencia intrínseca del esfínter (13).
Como ya hemos encontrado en estudios previos en nuestro centro (16), las principales complicaciones con el TVT son de tipo irritativo (urgencia e incontinencia de urgencia), seguido de cerca por los síntomas obstructivos, mientras que estos con el TOT son mínimos. Se han reportado resultados similares de varios autores al respecto, por ello, Kyu-Sung y cols sugieren una hipótesis para explicar este fenómeno, basándose en que la orientación de la malla TOT al ser similar a una hamaca natural, disminuye el efecto obstructivo que aumentaría la resistencia uretral, reduciendo también los síntomas irritativos, y además, si existen problemas para el vaciado, suelen resolverse tras con cateterismo vesical por unos días, sin requerir otro tipo de intervenciones $(7,4,6,13,16,17)$.

La principal diferencia reportada entre los dos procedimientos es que al evadir el espacio retropúbico, disminuyen las lesiones vesicales. En nuestro estudio no hemos encontrado esta complicación para ninguna de las técnicas.

La principal limitación de nuestro estudio es no ser randomizado, sin embargo, las características de las pacientes intervenidas son comparables entre si. El tiempo de seguimiento es relativamente corto, pero podríamos decir que es suficiente para evaluar los resultados, ya que como lo hemos dicho anteriormente, la práctica totalidad de los fracasos suceden antes de los 6 meses tras la cirugía. No obstante, requeriremos de mayor tiempo de seguimiento para poder sustentar esta hipótesis.

Otra limitación de nuestro estudio, es la definición estricta de lo que consideramos fracaso, ya que el estudio de supervivencia no nos permite parámetros intermedios, teniendo que considerar como incontinente cualquier pérdida de orina, aun cuando la calidad de vida de la paciente halla mejorado.

TABLA II. COMPARACIÓN DE LAS COMPLICACIONES ENCONTRADAS CON LAS DOS TÉCNICAS EMPLEADAS.

\begin{tabular}{|c|cc|cc|}
\hline Complicaciones & \multicolumn{2}{|c|}{ TVT } & \multicolumn{2}{c|}{ TOT } \\
\hline Urgencia postoperatoria & 23 & $33,3 \%$ & 5 & $8,5 \%$ \\
\hline Incontinencia de urgencia & 13 & $18,8 \%$ & 6 & $10,2 \%$ \\
\hline Dolor postoperatorio & 3 & $4,3 \%$ & 12 & $20,3 \%$ \\
\hline Infección de la herida & 1 & $1,4 \%$ & 0 & $0 \%$ \\
\hline Retención de orina & 8 & $11,6 \%$ & 1 & $1,7 \%$ \\
\hline
\end{tabular}




\section{CONCLUSIONES}

Nuestros resultados con las técnicas de TVT y TOT para tratamiento de la incontinencia de esfuerzo femenina son similares, presentando fracasos principalmente durante los primeros meses tras el procedimiento, pudiendo predecir el comportamiento posterior, sin embargo, tendremos que esperar a tener un tiempo de seguimiento mas largo para aceptar dicha conclusión. Las complicaciones tipo urgencia miccional son significativamente mayores con el TVT.

\section{BIBLIOGRAFÍA y LECTURAS RECOMENDADAS (*lectura de interés $y^{* *}$ lectura fundamental)}

1. ASOCIACIÓN ESPAÑOLA DE UROLOGÍA Y SOCIEDAD ESPAÑOLA DE GINECOLOGÍA Y OBSTETRICIA.: "Tratado de incontinencia urinaria”. Luzán Editores, pag. 545, 2006.

2. ABRAMS, P.; CARDOZO, L.; FALL, M. y cols.: "The standardisation of terminology in lower urinary tract function: Report from the standardisation sub-committee of the international continence society". Urology, 61: 37, 2003.

*3. KELLY, H.; DUMM, W.M.: "Urinary incontinence in women without manifest injuty to the bladder: A report of cases". Surg. Gynecol. Obstet., 18: 444, 1914.

4. PAICK, J.S.; CHO, M.C.; OH, S.J. y cols.: "Factors influencing the outcome of mind urethral sling procedure for female urinary incontinence". J. Urol., 178: 985, 2007.

**5. ULMSTEN, U.; HENRIKSSON, L.; JOHNSON, P. y cols.: "An ambulatory surgical procedure under local anesthesia for treatment of female urinary incontinence". Int. Urogynecol., 7: 81, 1996.

*6. DETAYRAC, R.; DEFFIEUX, X.; DROUPY, S. y cols.: "A prospective randomized trial comparing tension-free vaginal tape and transobturator suburethral tape for surgical treatment of stress urinary incontinence". Am. J. Obstet. Gynecol., 190: 602, 2004.

7. KYU-SUNG, L.; DEOK HYUN, H.; YANG SU, C. y cols.: "A prospective trial comparing thension free vaginal taper and transobturator vaginal tape inside-out for surgical treatment of female stress urinary incontinence: 1-year follow up". J. Urol., 177: 214, 2007.

**8. DELORME, E.: "Transobturator urethral suspension: Mini-invasive procedure in the treatment of stress urinary incontinence in women". Prog. Urol., 11: 1306, 2001.

9. DARAI, E.; FROBERT, J.L.; GRISARD-ANAF, M. y cols.: "Functional results after the suburetral sling procedure for urinary stress incontinence: A prospective randomized multicentre stydy comparing the retropubic and transobturador routes". J. Eur. Urol., Article in press, 2006.

10. LUJAN, M.; PAEZ, A.; BUSTAMANTE, S. y cols.: "Análisis comparativo de diferentes técnicas para la corrección de la incontinencia de stress en la mujer. Revisión de nuestra experiencia”. Actas Urol. Esp., 21: 655, 1997.

11. REDONDO, E.; LUJAN, M.; PÁEZ, A. y cols.: "Análisis comparativo de complicaciones entre las diferentes técnicas empleadas para el tratamiento quirúrgico de la incontinencia urinaria de esfuerzo en nuestro medio". Arch. Esp. Urol., 56: 269, 2003.

12. KAPLAN, E.L.; MEIER, P.: "Nonparametric estimation from incomplete observations". J. Am. Stat. Assoc., 53: 457, 1958.

*13. ULMSTEN, U.; JOHNSON, P.; REZAPOUR, M.: "A three-year follow up of tension free vaginal tape for surgical treatment of female stress urinary incontinence". BJOG, 106: 345, 1999.

14. LIAPIS, A.; BAKAS, P.; GINER, M. y cols.: "Tension free vaginal tape versus tension free vaginal tape obturator in women with stress urinaty incontinence". Gynecol. Obstet. Invest., 62: 120, 2006.

15. MELLIER, G.; BENAYED, B.; BRETONES, S. y cols.: "Suburethral tape via transobturator route: Is the tot a simplification of the TVT?". Int. Urogynecol. J. Pelvic. Floor. Dysfunct., 15: 227, 2004.

16. TORRES, G.; LUJAN, M.; MARTÍN, C. y cols.: "Incontinencia de esfuerzo: Experiencia quirúrgica en nuestro centro". Actas Urol. Esp., 31: 858, 2007.

17. HOLMENGREN, C.; NILSSON, S.; LANNER, L. y cols.: "Frequency of the novo urgency in 463 women who had undergone the tension-free tape (TVT) procedure for genuine stress urinary incontinence. A long term follow up". J. Ejogrb., 5754, Article in press, 2006. 